\title{
Characterization of a thermoforming composite material made from hemp fibers and polypropylene
}

\author{
Emilia Ciupan ${ }^{1, *}$, Lucian Lăzărescu ${ }^{1}$, Ioan Filip ${ }^{2}$, Cornel Ciupan ${ }^{1}$, Emilia Câmpean $^{1}$, Ioan \\ Cionca $^{2}$ and Emanuela Pop $^{1}$ \\ ${ }^{1}$ Technical University of Cluj-Napoca, Memorandumului Street, No. 28, 400114 Cluj-Napoca, Cluj \\ County, Romania \\ ${ }^{2}$ TAPARO SA, 43562 Village Borcut, No. 98, Maramures County, Romania
}

\begin{abstract}
The paper refers to a composite material developed for manufacturing thermoformed products with applications in furniture making, automotive industry etc., a method and machinery for manufacturing the material in unwoven form. From this material, Research and Development Department of TAPARO SA has designed and built a series of furniture components. The composite material made of a thermoplastic fibrous component and hemp fibre component, the way of obtaining and the properties of the thermoformed material presented in the paper are necessary in the process of designing and optimizing the parts.
\end{abstract}

\section{Introduction}

The purpose of the paper is to present the mechanical properties of a composite material made by TAPARO SA Company and used for thermoforming of different furniture parts. The majority of the padded products contain a resistance structure in the form of a timber frame. Wood is an excellent material in terms of functional, environmental and aesthetic but its overexploitation has an impact on the environment, and most of the countries have adopted legislation regarding the forestry exploitation $[1,2]$. Therefore manufacturers that have large series of articles containing pieces of wood, including furniture manufacturers, seek alternatives of wood with other recyclable products that provide productivity and overall cost benefits of the product. In this regard were developed a series of composite materials made of natural fibers and thermoplastics, which enable the thermoforming manufacture of the parts previously made of wood. Replacing wooden products involves redesigning components, but the optimization of the project requires a series of physical and mechanical properties of the product: tensile strength, elastic modulus, shear modulus, mass density, Poisson' ratio etc. These properties vary depending on the component fibrous mixture and the thermoforming process, but the literature offers no concrete values.

Panaitescu [3] studied the thermal stability of hemp fibers when treated with different silane treatments. Polypropylene copolymer BJ380MO was mixed with maleic anhydride

\footnotetext{
*Corresponding author: emilia.ciupan@mis.utcluj.ro
} 
grafted polypropylene, Polybond 3200, and also with short cut hemp fibers with the length of about $2 \mathrm{~mm}$. Using an electrically heated press (Dr. Collin, Germany) were moulded at the dimension of $100 \times 100 \times 1 \mathrm{~mm}$ and then compressed, at a temperature of $180^{\circ} \mathrm{C}$.

The samples were noted as PP/HF (polypropylene/ hemp fibers) for the composite with untreated fibers and PP/HF-APS, PP/HF-MPS, PP/HF-GPS and PP/HF-KP for the composites containing HF modified with APS ( $\gamma$-Aminopropyltriethoxysilane), MPS ( $\gamma$ Methacryloxypropyltrimethoxysilane), GPS ( $\gamma$-Glycidoxypropyltrimethoxysilane) and KP (potassium permanganate), respectively. Using a Universal Testing Machine Instron 3382 tensile properties of the composites were determined for five specimens of each sample. The results concluded that when the amount of HF-MPS grown from $0 \mathrm{wt} \%$ to $40 \mathrm{wt} \%$ the PP reinforced with HF-MPS has an increase of modulus compared to the composites containing untreated fibers. The increase was with $67 \%$ in PP/40 wt $\% \mathrm{HF}-\mathrm{MPS}$ and with only $30 \%$ when PP was reinforced with the same amount of untreated fibers.

Also, nanoindentation tests were performed. Using the Oliver-Pharr method, hardness and elastic modulus were determined. The results shown different hardening effect of treated HF, better for KP, MPS and MAPP (maleated polypropylene) treatments, intermediate for APS and worse for GPS, similar to macro-level mechanical results. Also, the results showed that TM (tensile modulus of elasticity) increased with $47 \%$ and $31 \%$ for the composites with MAPP and KP treatments, and with $26 \%$ and $37 \%$ for NM (nanoindentation modulus).

Z.L. Yan [4] studied the difference in morphology, composition and thermal resistance of noil fibre for the fabrication of injection moulded NFRC (natural fibre reinforced composites). The results were compared with scutched hemp fibre (SHF). The tensile, flexural and impact strengths of composites with both kinds of fibre are compared with different fibre and maleic anhydride polypropylene (MAPP) addition content. Theresa Sullins [5] studied also the effects of the fiber chemical treatment and/or polymer matrix treatment with coupling agent, on the mechanical properties of the hemp fiber polypropylene matrix composites. The study used different fiber length during the processing. Another study that used hemp as raw material was the study of M.R. Bambach [6] how studied the intrinsic compression behaviour of natural fibre composites. For the study, composite plates from flax, jute and hemp fibre-resin were fabricated having the width of $150 \mathrm{~mm}$ and $450 \mathrm{~mm}$ length, with 2,3 or 4 fiber layers for the flax and jute channels, and 3, 4 and 6 fibre layers for the hemp channels.

Although the literature offers articles on the thermoforming of fibrous composite materials, it does not offer concrete values related to this process. The mechanical properties of this composite material, like specific stiffness and strength, have not been studied sufficiently and the producers of such materials do not provide concrete values.

\section{Composite materials and the manufacturing process}

\subsection{Composite material}

The majority of upholstered products have a structure in the form of a wood frame. For ecological reason, a series of composite materials made of natural and thermoplastic fibers have been developed, materials which can be thermoformed so as to replace products made of wood.

Patent KR970008215, "Thermoplastic composite material reinforced with hemp fibers", refers to a composite material made of a thermoplastic reinforced with hemp fibers and filler represented by wood. The thermoplastic is represented by polypropylene, polyethylene, a copolymer of ethylene and polypropylene, a copolymer of acrylonitrile - 
butadiene-styrene or simply nylon. The composite is manufactured as sheets used in diecutting or pellets used in injection moulding.

Another patent FR2781492 „Composite thermoplastic material for use in production of various moulded articles includes hemp fibers of specified dimensions and humidity" refers to a thermoplastic composite which includes hemp fibers of sizes and humidity fit for moulded products. The composite material is formed from thermoplastic material, with a maximum melting point of $200^{\circ} \mathrm{C}$, and hemp fibers shorter than $2 \mathrm{~mm}$ with a diameter smaller than or equal to $0.2 \mathrm{~mm}$. The patent describes a method of manufacturing the material consisting in melting the thermoplastic and mixing hemp fibers into it.

The disadvantage of the material obtained by the patented method consists of the fact that it has small strength due to the short fibers and is recommended to be used in injection moulding and less for thermoforming.

The composite material for thermoforming, which will be characterized in this work, is made of a thermoplastic fibrous component consisting of 20-60 mm long and 7-16 DEN fine polypropylene fibers representing $40 \%$ to $50 \%$ of the total material weight, and a plant fiber component which can be hemp who has 70-80 DEN fine and 60 to $100 \mathrm{~mm}$ in length and represents $50 \%$ of the total material weight.

\subsection{The manufacturing process and the machinery}

The manufacturing process of the composite material consists of the following operations:

a. taking the plant fibers from the bale and cutting them to lengths between 5 and $100 \mathrm{~mm}$, using a rotating blade chopping machine;

b. simultaneous weighing of the plant fibers resulted from the previous phase in order to obtain a mix for the composite material of which the plant fibers represent $50-60 \%$ of the total mass;

c. coarse mixing of the plant and polypropylene fibers and defibering them with the help of a fiber opener with nails;

d. mixing and finely shredding the materials;

e. interlacing the material and reducing its thickness by a factor of 4 to 5

f. pulling and rolling the material in order to make a fabric with consolidated fibers.

The machinery for producing the composite material (Fig. 1) consists of at least two feeding modules, one for the thermoplastic fibers (1) and the other for the plant fibers (2), one module (3) which weighs and feeds correct proportions of each type of fiber, one module (4) for the primary mixing and the coarse defibering, one module (5) for the fine mixing, one module (6) for defibering, one module (7) for interlacing and one module (8) for pulling and rolling the material.

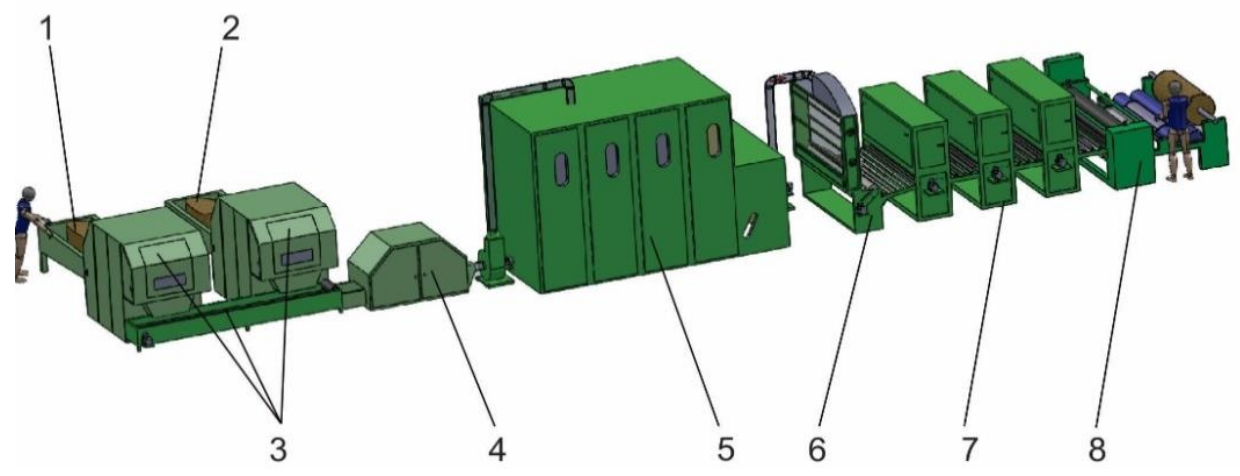

Fig. 1. The modular structure of the machinery for the manufacturing of the composite material. 


\subsection{The samples}

From the nonwoven material, plates of $350 \times 350 \mathrm{~mm}$ were cut and then packages were made, as shown in Table 1. With $\mathrm{L}$, the feed direction of the material to the interlacing machines was noted, and with $\mathrm{T}$, a direction perpendicular to it. From the materials presented in Table 1, plates were thermoformed. The thermoformed process was carried out in two stages:

a. Heat the material at $220{ }^{\circ} \mathrm{C}$ using a heated plate press.

b. Simultaneous pressing of 5 plates on a $600 \mathrm{kN}$ press.

The plates were numbered according to the number of layers and layer placement direction.

Table 1. Making the sheet.

\begin{tabular}{|l|c|c|c|}
\hline No. & $\begin{array}{c}\text { No. of } \\
\text { layers }\end{array}$ & Layout & No. of sheet \\
\hline 1. & 3 & L-L-L & 5 \\
\hline 2. & 3 & L-T-L & 5 \\
\hline 3. & 4 & L-L-L-L & 5 \\
\hline 4. & 4 & L-T-L-T & 5 \\
\hline
\end{tabular}

From the heat-treated plates (Table 1), with a guillotine, 110 samples with the dimensions of $250 \times 25 \mathrm{~mm}$ were cut (Fig. 2, Table 2).

\section{Performing the tests and composite material characterization}

\subsection{Testing machine}

The Zwick Roell Z150 was used to perform the tensile tests. The samples and test methods were performed according to the standard D3039, „Test Method for Tensile Properties of Polymer Matrix Composite Materials".

The machine used is an electromechanical one, having a kinematic axis with a leading screw.

The machine control, the data acquisition, as well as the processing and export of results are done using the computer through a program called testXpert II.

The test was done having the following machine settings:

- grip to grip separation at the start position $150 \mathrm{~mm}$;

- pre-load -0,4 MPa;

- test speed $-2 \mathrm{~mm} / \mathrm{min}$.

As said before, from the thermoformed plates (Table 1) 110 samples with the dimensions of $250 \times 25 \mathrm{~mm}$ were cut (Table 2). All the samples from Table 2 were labelled, as shown in Figure 2.

Table 2. Test samples.

\begin{tabular}{|c|c|c|c|}
\hline No. & No. of layers & Layout of & No. of \\
\hline 1. & \multirow{3}{*}{3 (L-L-L) } & $\mathrm{L}$ & 10 \\
\hline 2. & & $\mathrm{~T}$ & 10 \\
\hline 3. & & D & 10 \\
\hline 4. & \multirow{3}{*}{3 (L-T-L) } & $\mathrm{L}$ & 10 \\
\hline 5. & & $\mathrm{~T}$ & 10 \\
\hline 6. & & $\mathrm{D}$ & 10 \\
\hline 7. & \multirow{3}{*}{4 (L-L-L-L) } & $\mathrm{L}$ & 10 \\
\hline 8. & & $\mathrm{~T}$ & 10 \\
\hline 9. & & $\mathrm{D}$ & 10 \\
\hline 10. & \multirow[t]{2}{*}{4 (L-T-L-T) } & $\mathrm{L}$ & 10 \\
\hline 11. & & $\mathrm{D}$ & 10 \\
\hline
\end{tabular}



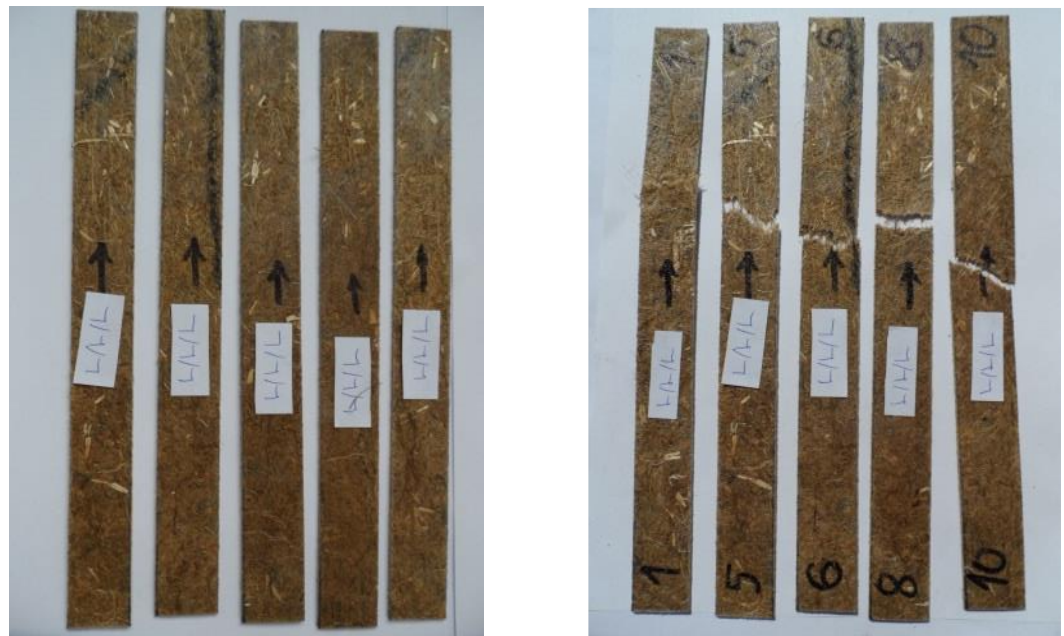

Fig. 2. Samples before and after the tests.

\subsection{Results}

The results of the tests are shown graphically in the figures from Table 3 . In Table 3 the following notations were used: $\sigma \mathrm{M}$ - tensile strength, $\varepsilon \mathrm{M}-$ strain at tensile strength, $\sigma \mathrm{B}-$ stress at break, $\varepsilon \mathrm{B}-$ strain at break.

Table 3. The stress-strain diagrams

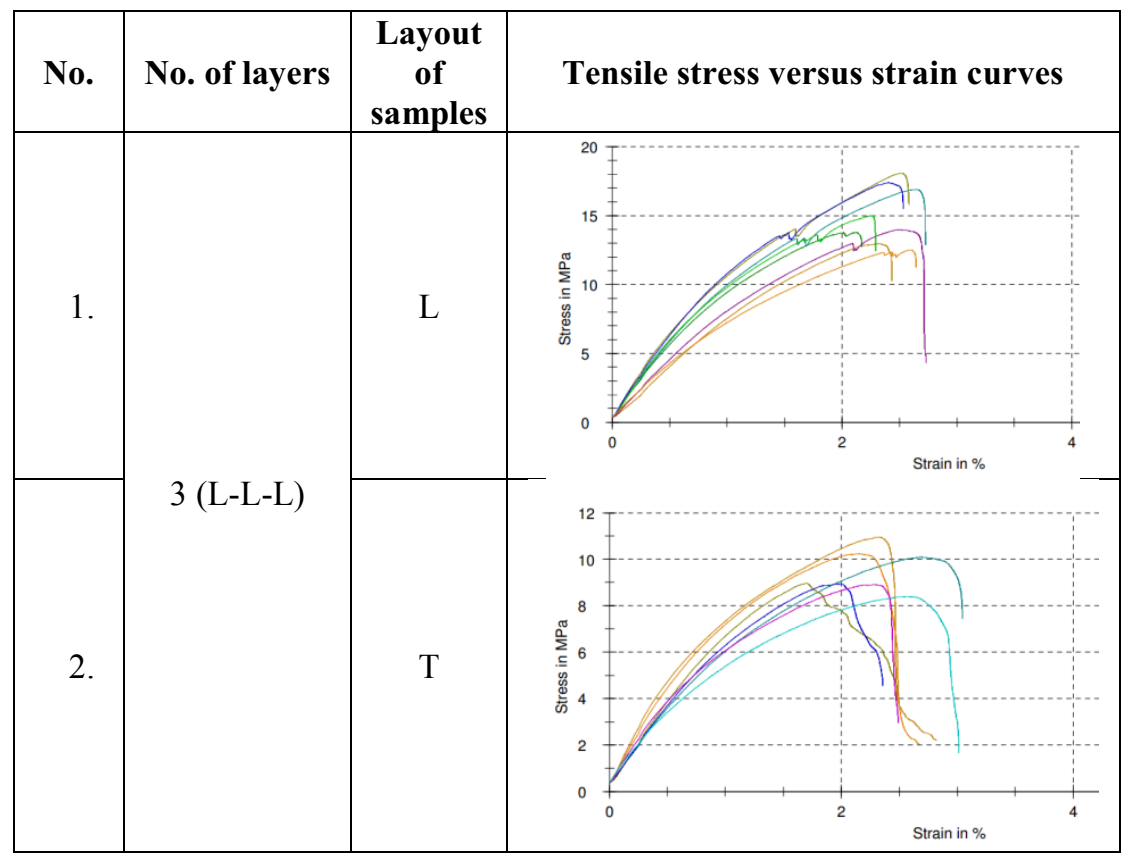




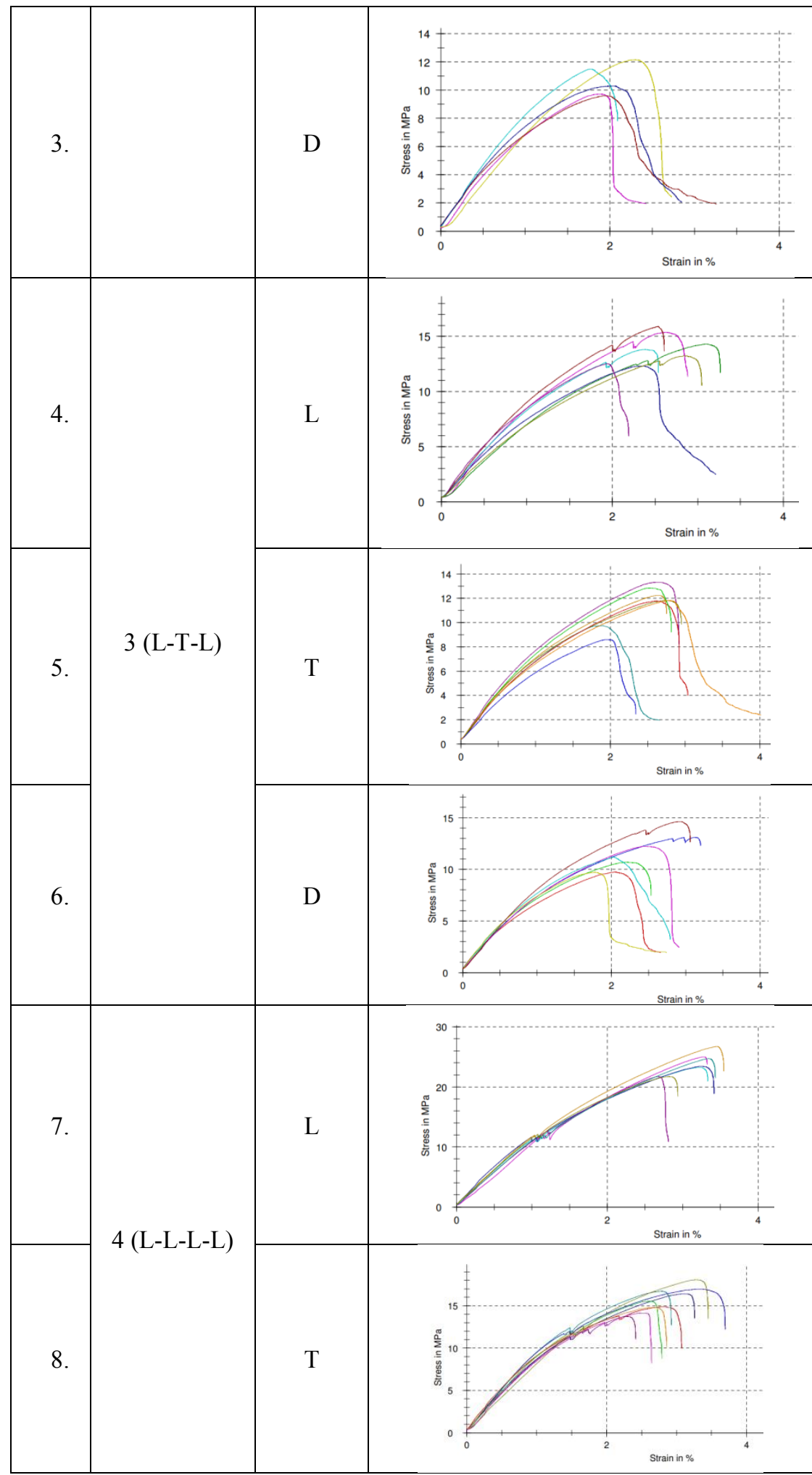




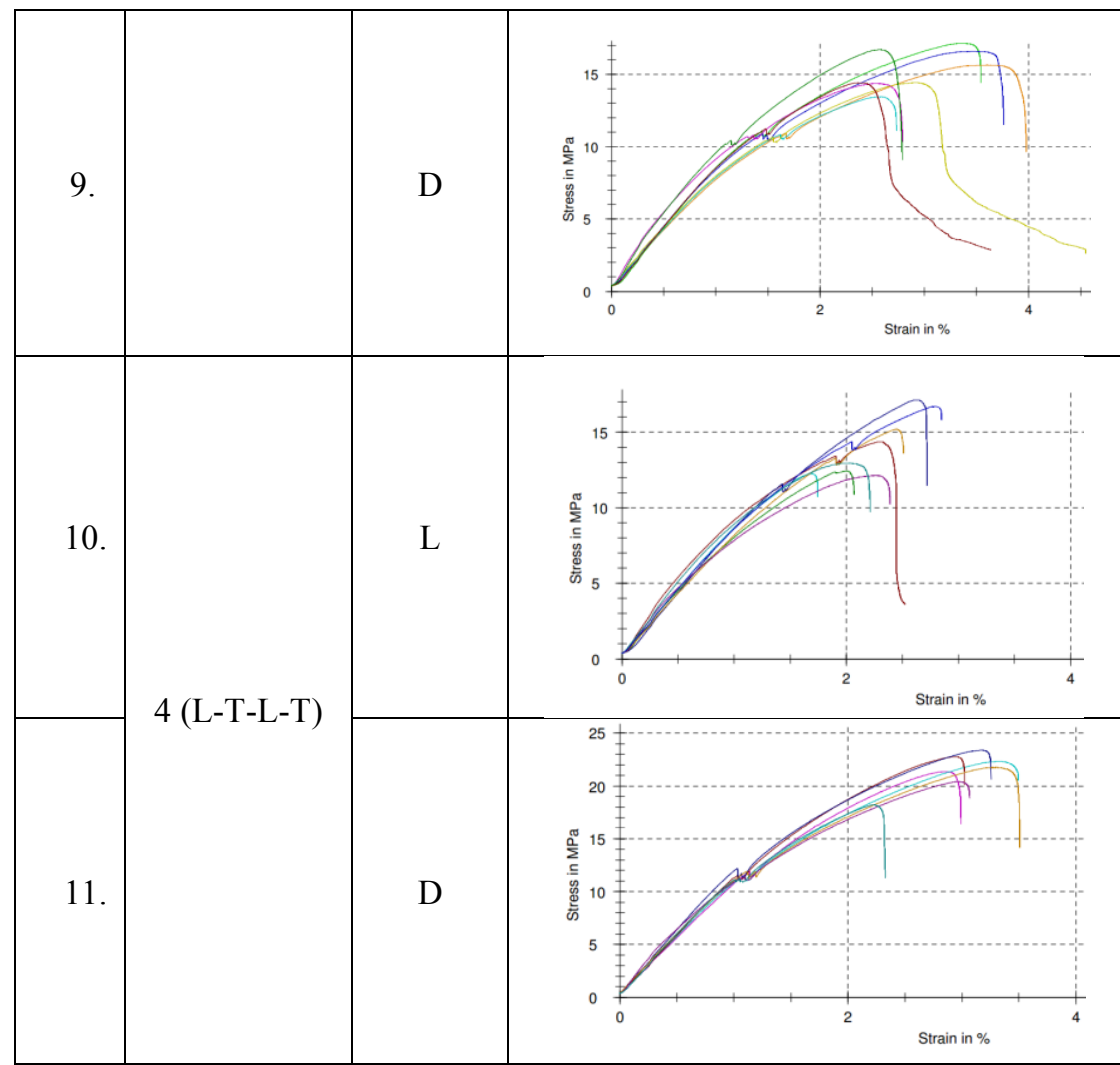

The mean values of the tests are summarized in Table 4. After performing the tests, a smoothing of the results was made by removing samples that gave extreme values. In columns 3-6 are presented the mean values of a set of 7 samples for each layout of samples ( $\mathrm{L}$ - longitudinal, $\mathrm{T}$ - transversal, D - diagonal), and in columns 7-10 were calculated the mean values for all samples Layout of layers from column 1.

Table 4. The tensile properties of thermoformed composite material.

\begin{tabular}{|c|c|c|c|c|c|c|c|c|c|}
\hline \multirow{2}{*}{$\begin{array}{l}\text { Layout of } \\
\text { layers }\end{array}$} & \multirow{2}{*}{$\begin{array}{c}\text { Layout of } \\
\text { samples }\end{array}$} & \multicolumn{4}{|c|}{ Average values - Layout of samples } & \multicolumn{4}{|c|}{ Average values - Layout of layers } \\
\hline & & $\sigma_{\mathbf{M}}[\mathrm{MPa}]$ & $\varepsilon_{\mathbf{M}}[\%]$ & $\sigma_{\mathrm{B}}[\mathrm{MPa}]$ & $\varepsilon_{\mathrm{B}}[\%]$ & $\sigma_{\mathbf{M}}[\mathbf{M P a}]$ & $\varepsilon_{\mathbf{M}}[\%]$ & $\sigma_{\mathrm{B}}[\mathrm{MPa}]$ & $\varepsilon_{B}[\%]$ \\
\hline 1 & 2 & 3 & 4 & 5 & 6 & 7 & 8 & 9 & 10 \\
\hline \multirow{3}{*}{ LLL } & L & 15.1 & 2.4 & 15.1 & 2.4 & \multirow{3}{*}{11.77} & \multirow{3}{*}{2.20} & \multirow{3}{*}{9.53} & \multirow{3}{*}{2.27} \\
\hline & $\mathrm{T}$ & 9.5 & 2.2 & 7.58 & 2.4 & & & & \\
\hline & D & 10.7 & 2 & 5.91 & 2 & & & & \\
\hline \multirow{3}{*}{ LTL } & L & 13.9 & 2.5 & 12.5 & 2.6 & \multirow{3}{*}{12.33} & \multirow{3}{*}{2.47} & \multirow{3}{*}{10.04} & \multirow{3}{*}{2.60} \\
\hline & $T$ & 11.5 & 2.5 & 9.36 & 2.5 & & & & \\
\hline & $\mathrm{D}$ & 11.6 & 2.4 & 8.26 & 2.7 & & & & \\
\hline \multirow{3}{*}{ LLLL } & L & 23.8 & 3.2 & 23.8 & 3.2 & \multirow{3}{*}{18.30} & \multirow{3}{*}{2.97} & \multirow{3}{*}{17.77} & \multirow{3}{*}{3.00} \\
\hline & $\mathrm{T}$ & 15.4 & 2.9 & 13.8 & 3 & & & & \\
\hline & D & 15.7 & 2.8 & 15.7 & 2.8 & & & & \\
\hline \multirow{2}{*}{ LTLT } & $\mathrm{L}$ & 16.7 & 2.9 & 16.7 & 2.9 & \multirow{2}{*}{19.10} & \multirow{2}{*}{2.95} & \multirow{2}{*}{19.10} & \multirow{2}{*}{2.95} \\
\hline & D & 21.5 & 3 & 21.5 & 3 & & & & \\
\hline
\end{tabular}




\section{Conclusions}

The following conclusions are drawn from the conducted tests regarding the mechanical characteristics of the thermoformed material:

a. The thermoformed material made by overlapping multiple layers of reinforced nonwoven provides a higher $\sigma_{M}$ mechanical strength. This is due to a better homogenize of the plant fibers and of the thermoplastic mixture. Thermoformed samples obtained by overlaying four layers give 55\% more resistance than samples obtained by overlaying three layers.

b. Tensile strength varies depending on the layering of nonwoven fabrics. Thus, the best resistance is obtained in the direction of the feed direction (L) and the smallest in the perpendicular direction to it $(\mathrm{T})$. At cross-sectional stress, the residual decreases by approximately $40 \%$ for the samples obtained by overlapping layers in the same direction (L).

c. The samples cut diagonally (D) at $45^{\circ}$ to the $\mathrm{L}$ or $\mathrm{T}$ directions have higher resistance than the transverse ones, but close to it for the LLL and LLLL layers. When laying LTLT layers, the diagonal stress presents the best resistance $\left(\sigma_{\mathrm{M}}=21.5 \mathrm{MPa}\right)$.

d. Cross-overlay of non-woven (LTL; LTL) layers increases the minimum strength (Tdirection) and medium resistance by approximately $5 \%$.

e. Tensile strength at break $\sigma_{\mathrm{B}}$ increases with the number of layers and is approaching the maximum tensile strength $\sigma_{\mathrm{M}}$.

f. Strain at tensile strength has approximately the same value in the maximum breaking point.

\section{References}

1. I. Filip, E. Ciupan, I. Cionca, M. Ciupan, E. Pop, E. Campean, V. Heres, F. Raţ, C. Gherghel, The $21^{\text {th }}$ International Conference of Inventics, Iasi, 38-45 (2017)

2. M. Steopan, C. Ciupan, E. Campean, E. Pop, I. Filip, L Stelea. The $21^{\text {th }}$ International Conference of Inventics, Iasi, 95 - 103 (2017)

3. D. M. Panaitescu, C. A. Nicolae, Z. Vuluga, C. Vitelaru, C. G. Sanporean, C. Zaharia, D. Florea, G. Vasilievici, J. of Industrial and Engineering Chemistry, 37, 137-146 (2016)

4. Z.L. Yan, H. Wanga, K.T. Lau, S. Pather, J.C. Zhang, G. Lin, Y. Ding, Composites: Part B, 46, 221-226 (2013)

5. T. Sullins, S. Pillay, A. Komus, H. Ning, Composites Part B, 114, 15-22 (2017)

6. M.R. Bambach. Compression strength of natural fibre composite plates and sections of flax, jute and hemp. Thin-Walled Structures, 119, 103-113 (2017). 